Supporting Information for

\title{
Energy Decomposition Analysis of the Adhesive Interaction between an Epoxy Resin Layer and a Silica Surface
}

Chisa Higuchi and Kazunari Yoshizawa*

Institute for Materials Chemistry and Engineering and IRCCS, Kyushu University, Nishi-ku, Fukuoka 819-0395, Japan

*To whom correspondence should be addressed.

E-mail: kazunari@ms.ifoc.kyushu-u.ac.jp

Table of contents of this PDF:

S1. Hydroxylation of the silica surface .....................................................................2

S2. Comparison of dispersion correction methods between D2, D3, and TS for the construction of the silica surface model ..........................................................................

S3. Modeling process of the Layer model ................................................................. 3

S4. Calculation accuracy of HF+D2 combination ........................................................ 3

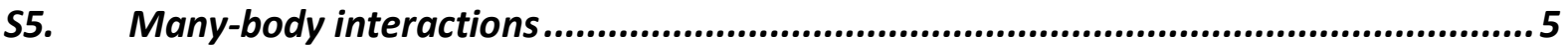

S6. Size effect of the clustered silica surfaces ............................................................... 7

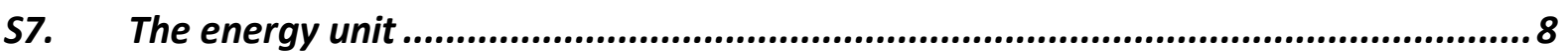

S8. Definition of the $3.6 \AA$ interacting region........................................................ 9

S9. Detailed analyses of the $3.6 \AA$ interacting region ............................................. 9

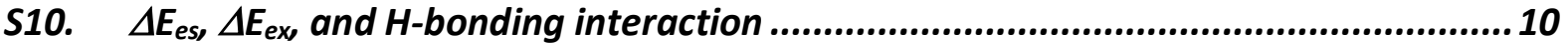

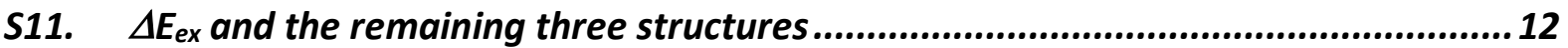

Other Supplementary Material for this manuscript includes the following:

Structures.zip

-xyz files of 20 cluster models from (a) to (t)

-xyz files of the Layer model and its initial structure (Figure S2(A) and (B)) 
Hydroxylation of the silica surface

The silica surface is known to be terminated with silanol groups $(-\mathrm{SiOH})$, which are assumed to be generated by the reaction with water in the air as shown in Figure S1. ${ }^{1}$

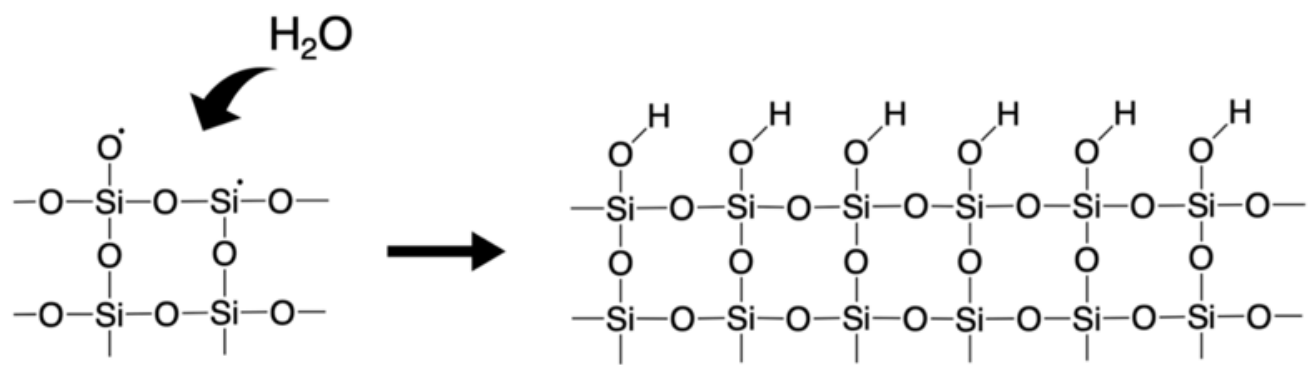

Figure S1. Schematic representation of the chemisorption process of water molecules onto a bare silica surface. Dots denote surface dangling bonds.

\section{S1. Comparison of dispersion correction methods between D2, D3, and TS for the construction of the silica surface model}

The bulk structure of $\alpha$-cristobalite fully optimized beforehand using density functional theory (DFT) calculations. We checked three types of dispersion corrections, D2-Grimme, D3Grimme, and Tkatchenko-Scheffler (TS) methods with the Vienna Ab initio Simulation Package (VASP) software. As shown in Table S1, the three methodologies showed almost the same optimized structures except the $\mathrm{Si}-\mathrm{O}-\mathrm{Si}$ angle. According to the experimental value ${ }^{3}$ in the first line, D2 and D3 methods are more appropriate for the silica surface modeling than the TS method. Between D2 and D3 method, we found no big differences. In this study, we finally chose D2-Grimme by following our previous work.²

Table S1. Lengths of Si-O bonds and angles of $\mathrm{O}-\mathrm{Si}-\mathrm{O}$ or $\mathrm{Si}-\mathrm{O}-\mathrm{Si}$ in the optimized structures using DFT-D2, DFT-D3, DFT-TS methods with VASP software. Relevant experimental values are shown in the first line. ${ }^{3}$

\begin{tabular}{ccccccccccc}
\hline & \multicolumn{3}{c}{ lengths $/ \AA$} & \multicolumn{4}{c}{ Angles $/{ }^{\circ}$} & \multicolumn{3}{c}{ lattice parameters } \\
\hline & Si-O & Si-O & O-Si-O & O-Si-O & O-Si-O & O-Si-O & Si-O-Si & a & b & c \\
\hline Experiment & 1.603 & 1.603 & 111.4 & 110.0 & 109.0 & 108.2 & 146.5 & - & - & - \\
D2-Grimme & 1.625 & 1.624 & 108.6 & 111.9 & 108.6 & 111.9 & 145.8 & 5.03 & 5.03 & 7.01 \\
D3-Grimme & 1.624 & 1.625 & 108.5 & 111.9 & 108.5 & 111.9 & 145.8 & 5.03 & 5.03 & 7.01 \\
TS & 1.624 & 1.626 & 108.9 & 111.9 & 108.9 & 111.9 & 140.4 & 4.94 & 4.94 & 6.81 \\
\hline
\end{tabular}


(A)

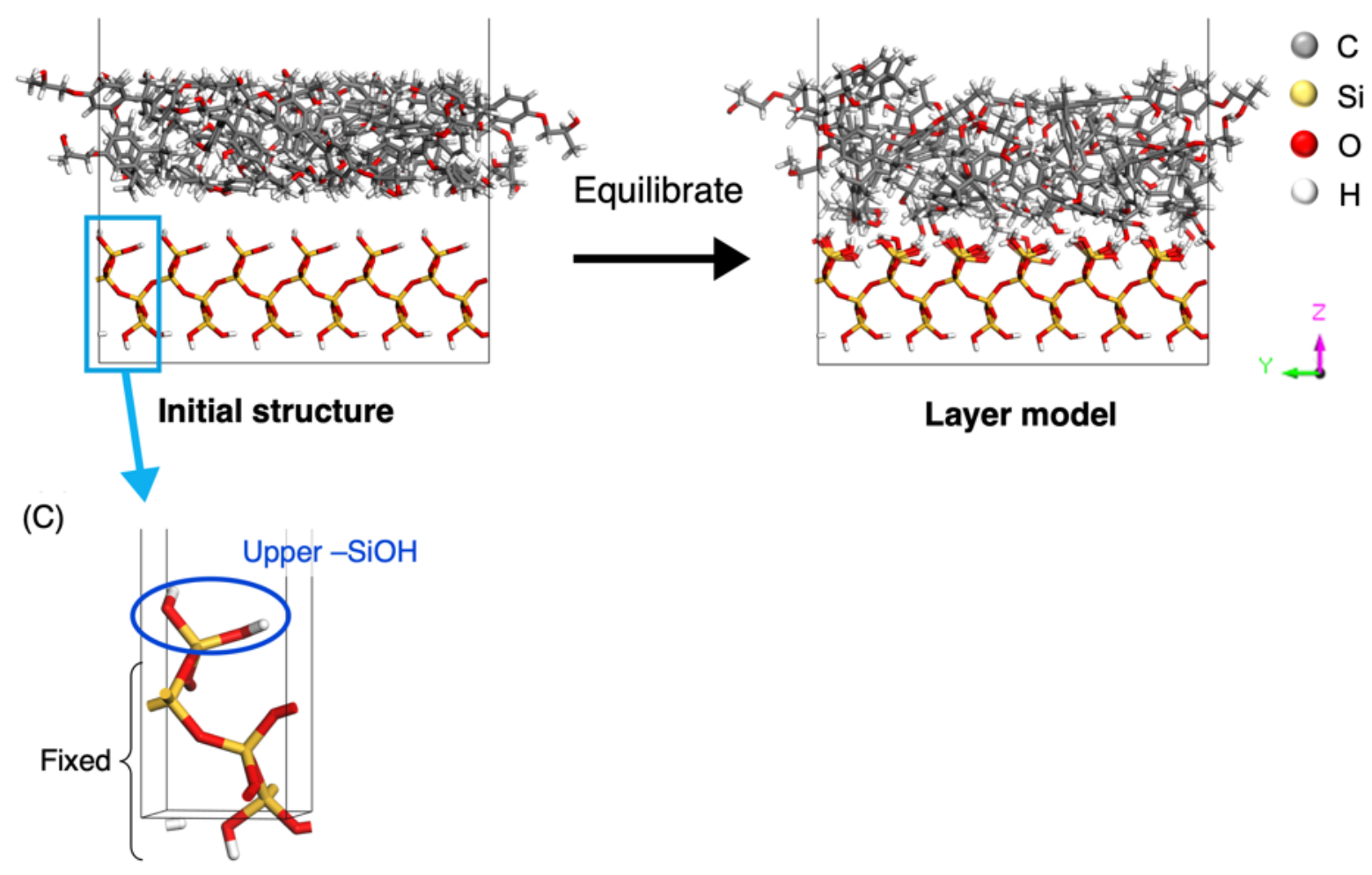

Figure S2. Adhesion interface equilibration. (A) Initial structure for the quench dynamics and (B) the layer model selected among 1000 optimized structures. (C) The upper silanol groups were allowed to move during the quench dynamics.

\section{S3. Calculation accuracy of $\mathrm{HF}+\mathrm{D} 2$ combination}

Currently, HF is used for PIEDA by following the methodology of our previous PIEDA work on a small epoxy/silica fragment model $^{4}$ to check the validity of our present results. For the dispersion correction, we used D2-Grimme method to keep the consistency with the methodology for the silica surface modeling (DFT+D2, as discussed in section S2). However, the D2 method is designed for DFT and not for HF, so that the combination is not perfectly appropriate. Therefore, we compared the HF+D2 results with DFT(GGA-PBE)+D2 results to check the accuracy of the HF+D2 calculation. Unfortunately, we do not have optimized structures of the Layer model or 20 cluster models with DFT(GGA-PBE)+D2, either because the layer model is too large to calculate with DFT, or because we do not have periodic model 
relevant to the 20 cluster models. Instead, we introduce our preliminary calculations for the model between a fragment of polyethylene terephthalate (PET) and hydroxylated silica surface (unpublished data) to check the accuracy of HF+D2 method. Figure S3(A) shows the structure of PET fragment model. The fragment contains a benzene group and three ester groups, which can form H-bonding interactions with the silica surface. $\Delta E_{\text {int }}$ and $\Delta E_{\text {disp }}$ between PET fragment and silica surface were estimated with both periodic-DFT (GGA-PBE+D2) and PIEDA $(\mathrm{HF}+\mathrm{D} 2)$ methods. Figure S3(B) and (C) show periodic and cluster models of PET/Silica interface for DFT and PIEDA calculations. As a result, the difference of the dispersion ratio contribution between $\mathrm{HF}+\mathrm{D} 2$ results and $\mathrm{DFT}+\mathrm{D} 2$ results was 0.04 as shown in Table S2.

(A)

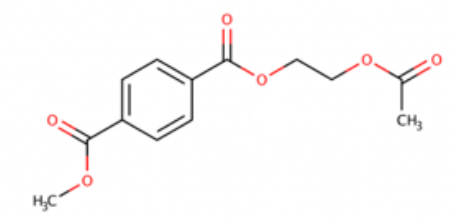

(B)
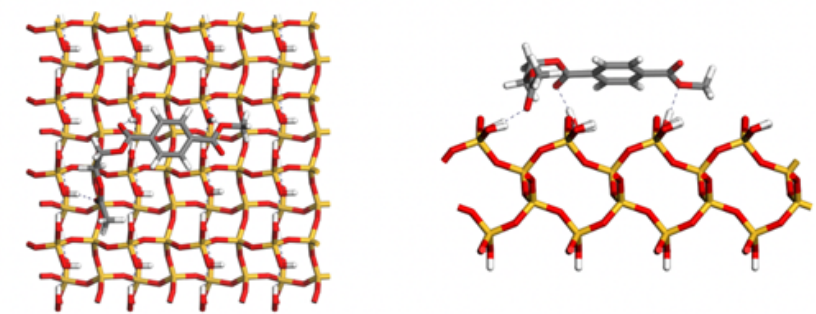

(C)

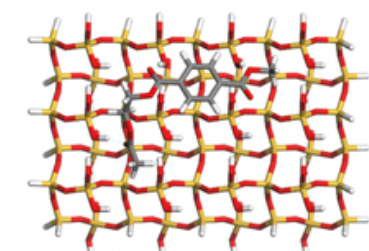

Top view

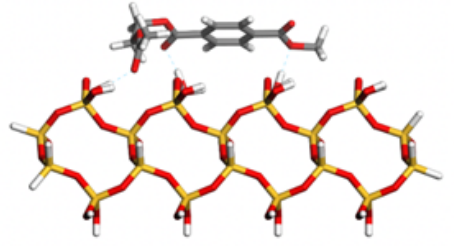

Side view

Figure S3. (A) the structural formula of PET fragment model, (B) periodic model for DFT(GGA-PBE)+D2 method, and (C) cluster model for HF+D2 (for PIEDA).

Table S2. Comparison of the dispersion (D2-Grimme) contribution to $\Delta E_{\text {int }}$ calculated with DFT and HF calculations. Energies are shown in $\mathrm{kcal} / \mathrm{mol}$.

\begin{tabular}{cccc}
\hline & $\Delta E_{\text {int }}$ & $\Delta E_{\text {disp }}$ & $\Delta E_{\text {disp }}$ ratio \\
\hline $\mathrm{DFT}+\mathrm{D} 2$ & 44.2 & 23.0 & 0.52 \\
$\mathrm{HF}+\mathrm{D} 2$ & 62.0 & 29.6 & 0.48 \\
\hline
\end{tabular}




\section{S4. Many-body interactions}

A cluster model (Hereafter Layer-cluster) was randomly cut out from the Layer model to investigate the effects of many-body interactions on the $\Delta E_{\text {int. }}$ The Layer-cluster model was sized appropriately to caluclate the entire system by using PIEDA. It contains 609 atoms with a size of $15 \times 18 \times 22 \AA^{3}$ and a polymer thickness of $13 \AA$ as shown in Figure S4. The two epoxy fragments shown in Figure S5 compose the epoxy layer of the Layer-cluster model.
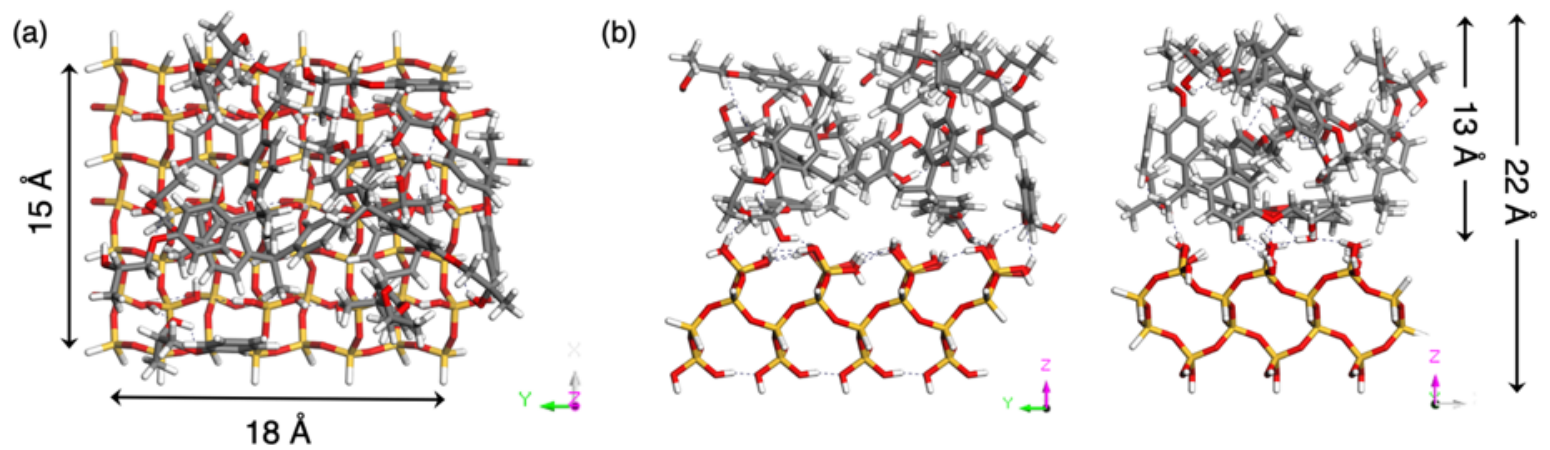

Figure S4. (a) Top and (b) side views of Layer-cluster model cut out of the periodic model for the PIEDA calculations.

(a)<smiles>CC(O)COc1ccc(C(C)(C)c2ccc(OCC(C)O)cc2)cc1</smiles>

(b)<smiles>CC(O)COc1ccccc1</smiles>

Figure S5. (a) The epoxy molecule and (b) the epoxy fragment whose size is almost the half of the epoxy molecule implemented into the epoxy layer in Layer-cluster model.

The many-body interaction is included in the PIEDA scheme. In Fedorov and Kitaura's PIEDA approach, the fragment molecular orbital (FMO) framework is adopted. ${ }^{5,6}$ In the FMO framework, the whole system is divided into fragments and pair interaction energies (PIEs) are calculated for each fragment pair. Since the electrostatic field from the whole system is considered in each individual fragment calculation, ${ }^{7}$ a PIEDA result originally includes manybody effect. On the other hand, to calculate PIEs without the many-body effect, we have also calculated individual epoxy fragment with the silica surface. In this case, the electrostatic field from the whole system does not include the surrounding epoxy fragments, so that the manybody effect can be eliminated. Figure S6a shows the model used for PIEDA with many-body 
effect. Each divided fragment is displayed in a different color, with numbers from 1 to 9. Figure S6b shows models used for PIEDA without many-body effect. Each PIEs calculated based on the nine different cluster models were summed to estimate the total PIE of the Layer-cluster model.

(a)

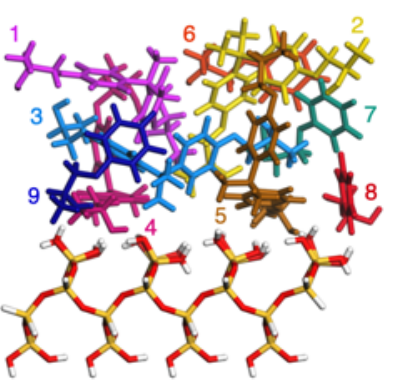

(b)

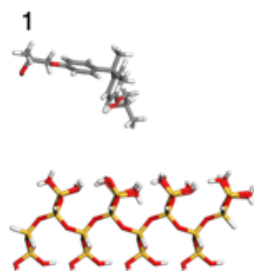

2
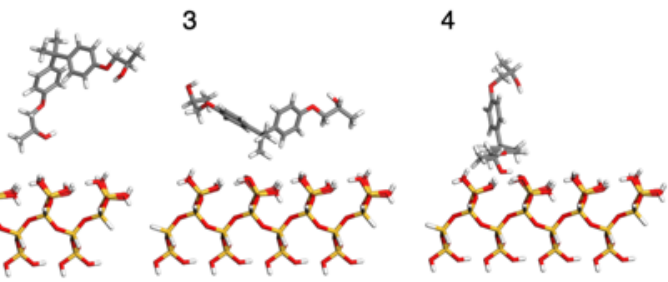

5

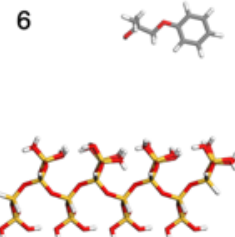

7

8

9
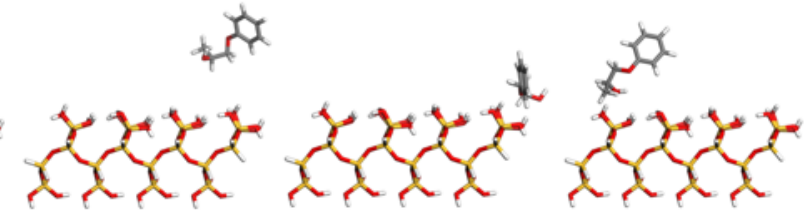

$z$
$y$

Figure S6. (a) PIEDA calculations with many-body effects for the Layer-cluster model (epoxy molecules are numbered from 1 to 9 , each with a different color). Fragments 1 to 5 are the epoxy molecules in Figure S5a and fragments 6 to 9 are the epoxy fragments in Figure S5b. (b) PIEDA calculation without many-body effects. Each epoxy molecule was placed individually on the silica surface.

Table S3 shows PIEs of each epoxy fragment calculated (a) with the many-body effect and (b) without the many-body effect. In the last column, the difference between (a) and (b) for each fragment is shown. Many-body effects can contribute to both stabilization and destabilization, and from the differences, we can recognize that fragments 1, 5, 6, and 9 were stabilized by the many-body effect, while fragments 2, 3, 4, and 8 were destabilized. However, in any cases, the differences of 9 fragments are very small within $1.6 \mathrm{~mJ} / \mathrm{m}^{2}$, and the sum shows no difference between (a) and (b). Therefore, we have concluded that the effect of many-body interaction on the $\Delta E_{\text {int }}$ is insignificant in the Layer-cluster model, and also in the Layer model. 
Table S3. Breakdown of $\Delta E_{\text {int }}\left(\mathrm{mJ} / \mathrm{m}^{2}\right)$ for each epoxy fragments from 1 to 9 in the Layercluster model (a) with and (b) without the many-body effect taken into account.

\begin{tabular}{cccc}
\hline Fragments & $\begin{array}{c}\text { (a) With many- } \\
\text { body effect }\end{array}$ & $\begin{array}{c}\text { (b) Without } \\
\text { many-body } \\
\text { effect }\end{array}$ & $\begin{array}{c}\text { Difference } \\
\text { (a) }-(\mathrm{b})\end{array}$ \\
\hline 1 & -0.7 & -0.5 & 0.2 \\
2 & 2.8 & 3.0 & -0.2 \\
3 & 17.7 & 17.8 & -0.1 \\
4 & 88.5 & 89.9 & -1.4 \\
5 & 96.9 & 95.2 & 1.6 \\
6 & -0.3 & -0.2 & 0.1 \\
7 & 0.5 & 0.5 & 0.0 \\
8 & 31.1 & 31.6 & -0.5 \\
9 & 51.9 & 51.1 & 0.9 \\
\hline SUM & 288.4 & 288.4 & 0.0 \\
\hline
\end{tabular}

\section{S5. Size effect of the clustered silica surfaces}

We have investigated the effect of silica surface size on PIEs. Figure S4 (a) shows the model $\mathrm{m}$ used in our study. The model in Figure S4 (b) is also based on the model m, but the silica surface was created smaller. As shown in Table S4, we compare PIEs of these two models to see the effect of silica surface size on the PIEDA results. Here, energies are shown in $\mathrm{kcal} / \mathrm{mol}$, which are originally obtained from PIEDA calculation, not $\mathrm{mJ} / \mathrm{m}^{2}$ because the silica surface area is not the same. The differences between (a) and (b) were very small in $\Delta E_{\text {itr }}$ and its energy components (within $3.1 \mathrm{kcal} / \mathrm{mol}$ ). Therefore, we have concluded that the effect of silica cluster size on the $\Delta E_{\text {int }}$ is insignificant in the 20 cluster models. 
(a)

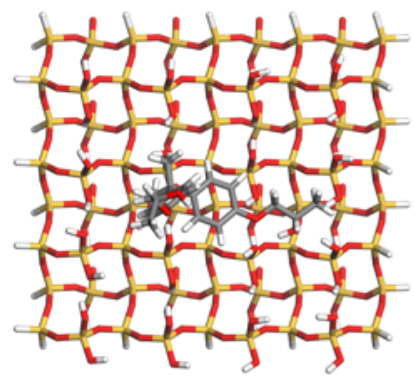

(b)

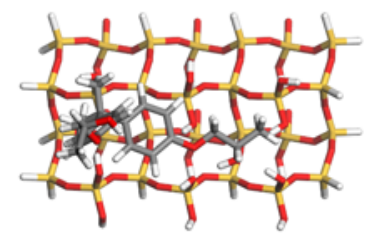

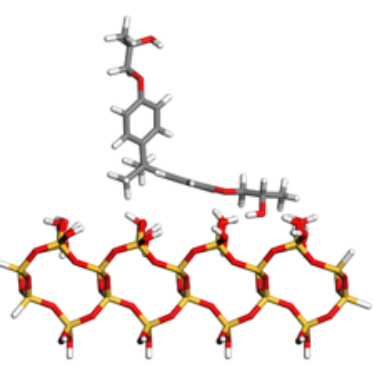

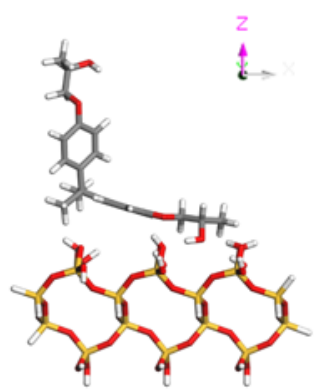

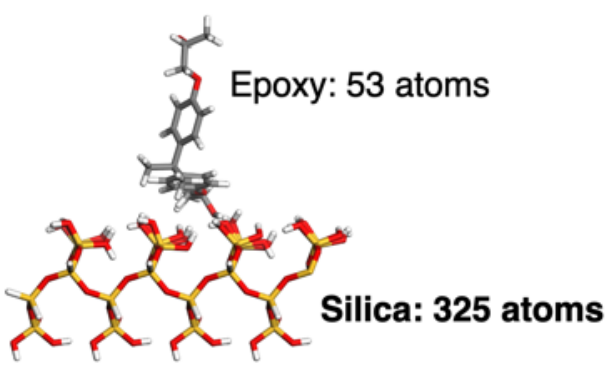

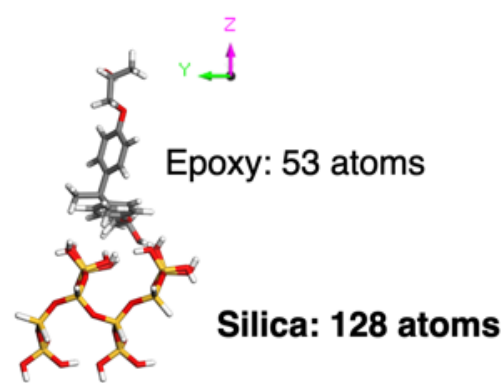

Figure S7. Models to check the effect of silica cluster size on PIEs. (a) The model $m$ used in the article and (b) the model newly created here based on the model $\mathrm{m}$ with smaller silica surface.

Table S4. PIEs in kcal/mol calculated from the two models in Figure S7

\begin{tabular}{cccccc}
\hline & $\Delta E_{\text {itt }}$ & $\Delta E_{\text {cs }}$ & $\Delta E_{\text {c }}$ & $\Delta E_{\text {ct }}$ & $\Delta E_{\text {dis }}$ \\
\hline (a) & 38.2 & 40.4 & -43.6 & 14.5 & 26.9 \\
\hline (b) & 35.1 & 39.0 & -43.4 & 14.4 & 25.1 \\
\hline Difference & 3.1 & 1.4 & -0.2 & 0.2 & 1.7 \\
\hline
\end{tabular}

\section{S6. The energy unit}

The unit $\mathrm{kcal} / \mathrm{mol}$ of PIEs were converted to $\mathrm{mJ} / \mathrm{m}^{2}$ by using the following equation:

$$
\Delta E_{\text {int }}\left(\mathrm{mJ} / \mathrm{m}^{2}\right)=\Delta E_{\text {int }}(\mathrm{kcal} / \mathrm{mol}) \times \frac{96.4853(\mathrm{~kJ} / \mathrm{mol})}{23.0609(\mathrm{kcal} / \mathrm{mol})} \times \frac{1}{N_{\mathrm{A}}} \times \frac{10^{6}}{a \times b}
$$

where $N_{\mathrm{A}}$ is Avogadro constant, $a$ and $b$ are the lengths of the adhesive area along the $x$ and $y$ axes. The lengths of $a=30 \AA$ and $b=30 \AA$, which are lattice parameters of the Layer model, 
were used in our study. The second term is for converting the units from $\mathrm{kcal} / \mathrm{mol}$ to $\mathrm{kJ} / \mathrm{mol}$. These numbers are energies of $1 \mathrm{eV}$ in units of $\mathrm{kJ} / \mathrm{mol}$ and $\mathrm{kcal} / \mathrm{mol}$, respectively.

\section{S7. Definition of the $3.6 \AA$ interacting region}

The 3.6 A interacting region was originally found by drawing a line between fragments 3, 4 5, 8, 9 and fragments 1, 2, 6, 7 in the Layer-cluster model. This border line was across the middle carbon atom of the epoxy molecule 5 circled by light blue color in Figure S8. The $\mathrm{z}$ coordinate of the carbon atom was $13.26 \AA$. The bottom line of the $3.6 \AA$ region was defined based on oxygen atoms of surface silanol groups of the silica surface. Since the surface of the Layer-cluster model does not align after the quench dynamics simulation, we referenced surface oxygen atoms in the initial structure before quench dynamics. The $\mathrm{z}$ coordinates of oxygen atoms of the surface silanol groups was $9.65 \AA$.

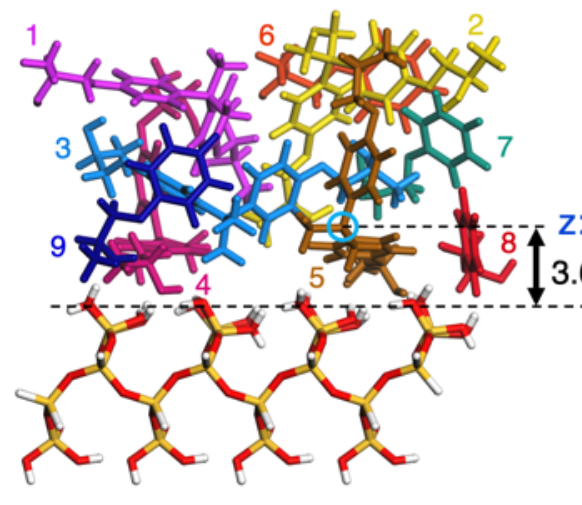

Layer-cluster model

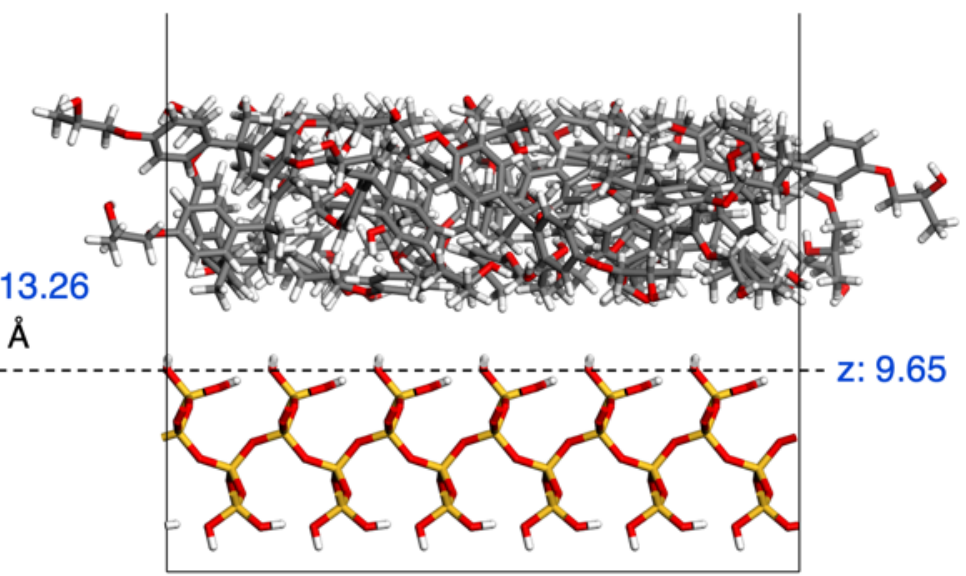

Initial structure

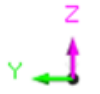

Figure S8. The z coordinates $(\AA)$ of top and bottom lines to define the $3.6 \AA$ interacting region.

\section{S8. Detailed analyses of the $3.6 \AA$ interacting region}

We investigated the 15 interacting molecules to determine which structural features result in their $\Delta E_{\text {es }}$ and $\Delta E_{\text {ex }}$ values. The structural features can be identified by comparing the results 
for similar structures. From section S9 to S11, we introduce detailed analyses for the remaining ten models that are not discussed in the manuscript.

The simplest similar pair is models (c) and (p); some of the epoxy molecules in both models are located near the $3.6 \AA$ border. As shown in Figure S9, the hydroxy group of model (c) is located in the $3.6 \AA$ region. This hydroxy group makes $\Delta E_{\text {es }}$ large and leads to a low $\Delta E_{\text {disp }}$ ratio (0.23). The methyl group of model (p), however, is located in the 3.6 Å region. The methyl group makes $\Delta E_{\text {disp }}$ large and results in a high $\Delta E_{\text {disp }}$ ratio (0.76). Model (p) is the only interacting model whose $\Delta E_{\text {disp }}$ ratio is affected by the $\Delta E_{\text {disp }}$ value directly.

(a)

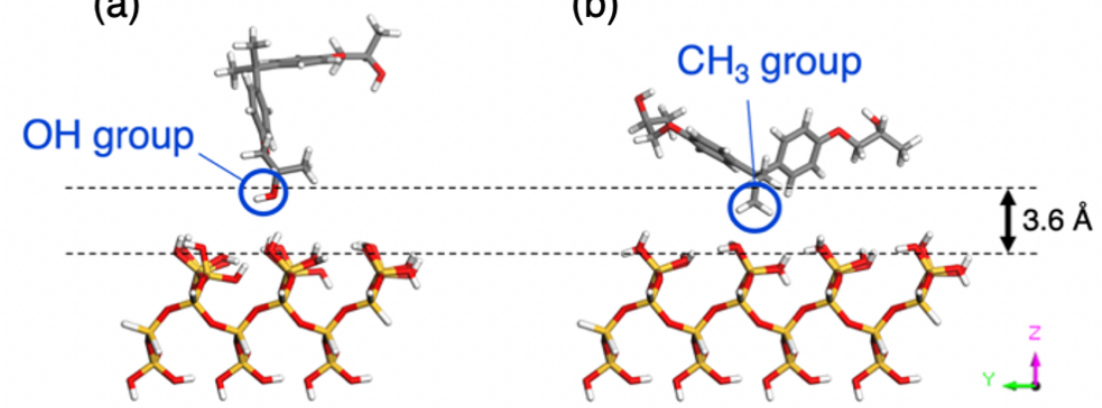

Figure S9. Comparison of models (a) c and (b) p. The region between the dashed lines with a distance of $3.6 \AA$ represents the interacting region.

\section{S9. $\Delta E_{\mathrm{es}}, \Delta E_{\mathrm{ex}}$, and $\mathrm{H}$-bonding interaction}

$\Delta E_{\text {ex }}$ is related to the difference in the $\Delta E_{\text {disp }}$ ratio between models (d) and (q). Their structures are similar in that the ends of epoxy molecules interact with the silica surface and form a single H-bond (Figure $\mathrm{S} 10$ ); however, the $\Delta E_{\text {disp }}$ ratio of model (d) is lower than the $W_{\text {ad }}{ }^{\mathrm{d}}$ ratio $(0.45)$ and the $\Delta E_{\text {disp }}$ ratio of model (q) is higher than the $W_{\text {ad }}{ }^{\text {d }}$ ratio $(0.77)$. A comparison of the energy differences of $\Delta E_{\mathrm{es}}, \Delta E_{\mathrm{ex}}, \Delta E_{\mathrm{ct}}$, and $\Delta E_{\mathrm{disp}}$ reveals that the $\Delta E_{\mathrm{ex}}$ of model (q) is $7.7 \mathrm{~mJ} / \mathrm{m}^{2}$ more negative than $\Delta E_{\text {ex }}$ of model (d), which is the largest difference between models (q) and (d) among the four $\Delta E_{\text {int }}$ components. This $\Delta E_{\text {ex }}$ difference is due to the distance between the epoxy molecule and the silica surface. Because $\Delta E_{\mathrm{ex}}$ is repulsive energy, the short distance between the epoxy molecule and silica surface makes $\Delta E_{\text {ex }}$ large and negative. The H-bonding distance of model (q) is certainly shorter (1.62 $\AA$ ) than the H-bonding distance of model (d) (1.71 $\AA$ ), as shown in Figure S10. The terminal methyl group of model 
(q) is also $\sim 0.5 \AA$ closer to the silica surface than that of model (d) according to the $z$-coordinate of each carbon atom in the methyl group. Nevertheless, the $\Delta E_{\text {disp }}$ values are approximately the same between models (d) and (q). All of these results are consistent with the sensitivity of $\Delta E_{\text {ex }}$ and $\Delta E_{\text {disp }}$ to the distance of the epoxy resin from the silica surface; the energy-distance curve for $\Delta E_{\mathrm{ex}}$ decreases rapidly as the epoxy resin is displaced farther from the silica surface, whereas the value of $\Delta E_{\text {disp }}$ decreases at a relatively slow rate. ${ }^{4}$ Thus, the epoxy-silica distance controls $\Delta E_{\mathrm{ex}}$, which differentiates the $\Delta E_{\text {disp }}$ ratios of models (d) and (q).
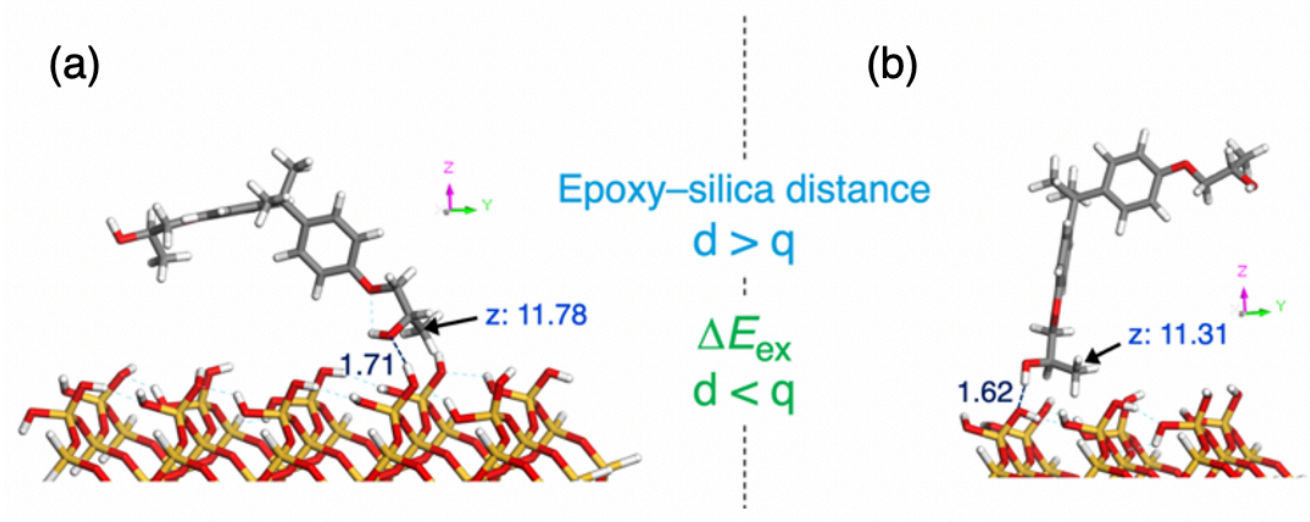

Figure S10. Comparison of models (a) $d$ and (b) q. The dashed blue lines represent the Hbonds formed between the epoxy molecule and silica surface. They are responsible for the interfacial interaction, the distances being expressed in $\AA$. The $z$-coordinates of the carbon atoms indicated by the black arrows are shown after " $z$ :" in units of $\AA$.

The $\Delta E_{\text {disp }}$ ratios of models (e), (f), and (n) vary because of the difference in the $\Delta E_{\text {es }}$ values. The epoxy molecules (e), (f), and (n) shown in Figure S11 are similar in that the epoxy ends interact with the silica surface. Furthermore, their structures are overall closer to the silica surface than the structures of models (d) and (q), resulting in the $\Delta E_{\text {int }}$ values of models (e) $\left(24.5 \mathrm{~mJ} / \mathrm{m}^{2}\right)$, (f) $\left(24.8 \mathrm{~mJ} / \mathrm{m}^{2}\right)$, and (n) $\left(19.1 \mathrm{~mJ} / \mathrm{m}^{2}\right)$ being $\sim 10 \mathrm{~mJ} / \mathrm{m}^{2}$ larger than the $\Delta E_{\text {int }}$ values of models (d) $\left(13.9 \mathrm{~mJ} / \mathrm{m}^{2}\right)$ and (q) $\left(8.7 \mathrm{~mJ} / \mathrm{m}^{2}\right)$. However, the ratios of $\Delta E_{\text {disp }}$ differ among the models. The $\Delta E_{\text {disp }}$ ratios of models (e) and (f) are lower than their $W_{\text {ad }}{ }^{\mathrm{d}}$ ratio, whereas the $\Delta E_{\text {disp }}$ ratio of model (n) is greater than its $W_{\text {ad }}{ }^{\mathrm{d}}$ ratio. With respect to energy differences $\Delta E_{\text {es }}, \Delta E_{\text {ex }}, \Delta E_{\text {ct, }}$ and $\Delta E_{\text {disp }}$, the largest difference is observed for $\Delta E_{\text {es }}$ between models (e) and (n) and between models (f) and (n). $\Delta E_{\text {es }}$ is mainly derived from H-bonding interactions. Indeed, there are two H-bonds in model (e) and three in model (f), whereas only 
one H-bond is formed in model (n) (Figure S11). Thus, the $\Delta E_{\text {disp }}$ ratios of models (e), (f), and (n) vary because of differences in $\Delta E_{\mathrm{es}}$, which arise from the number of $\mathrm{H}$-bonds.

(a)

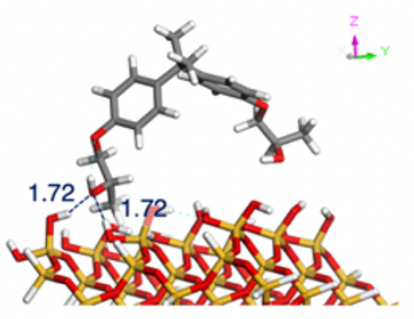

(b)

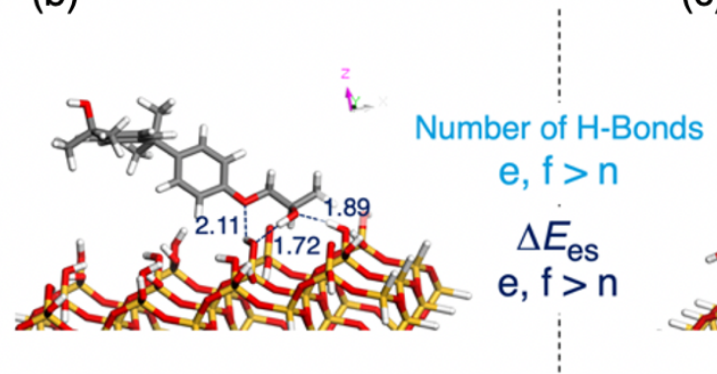

(c)

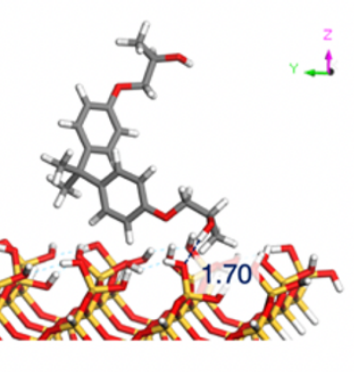

Figure S11. Comparison of models (a) e, (b) f, and (c) n. The blue dashed lines indicate the Hbonds formed between the epoxy molecule and the silica surface, which are responsible for the interfacial interaction, and the distance between them is shown in $\AA$.

\section{S10. $\Delta E_{\text {ex }}$ and the remaining three structures}

The remaining models among the 15 interacting models - (g), (i), and (k) — are similar to the five molecules discussed in the previous section in that one of the benzene rings, in addition to the terminal $\mathrm{OH}$ group, is close to the silica surface. However, $\mathrm{OH}-\pi$ interaction was not observed in these models. Irrespective of the commonalities, the $\Delta E_{\text {disp }}$ ratios of models (g) and (i) are lower than the $W_{\text {ad }}{ }^{\mathrm{d}}$ ratio (0.54 and 0.57), whereas that of model (k) is greater than the $W_{\text {ad }}$ ratio (0.65). In this case, $\Delta E_{\text {ex }}$, which originates from the position of the terminal $\mathrm{OH}$ groups, controls their $\Delta E_{\text {disp }}$ ratios. Figure $\mathrm{S} 12$ shows $z$-coordinates of the terminal $\mathrm{OH}$ groups of models (g), (i), and (k). Because the $\mathrm{OH}$ group of model (k) is closer to the silica surface than the $\mathrm{OH}$ groups of models (g) and (i), the $\Delta E_{\text {es }}$ value of model (k) $\left(44.0 \mathrm{~mJ} / \mathrm{m}^{2}\right)$ is greater than the $\Delta E_{\text {es }}$ values of models (g) $\left(33.7 \mathrm{~mJ} / \mathrm{m}^{2}\right)$ and (i) $\left(28.7 \mathrm{~mJ} / \mathrm{m}^{2}\right)$. However, more importantly, the $\Delta E_{\text {ex }}$ value of model $(\mathrm{k})\left(-46.9 \mathrm{~mJ} / \mathrm{m}^{2}\right)$ is negatively greater than the $\Delta E_{\text {ex }}$ values of models (g) and (i) $\left(-28.9 \mathrm{~mJ} / \mathrm{m}^{2}\right.$ and $-26.0 \mathrm{~mJ} / \mathrm{m}^{2}$, respectively), because of the proximity of the epoxy molecule to the silica surface. This proximity explains why the $\Delta E_{\text {disp }}$ ratios of models (g) and (i) are lower than their $W_{\text {ad }}{ }^{\mathrm{d}}$ ratio and why the $\Delta E_{\text {disp }}$ ratio of model (k) is greater than its $W_{\text {ad }}{ }^{\text {}}$ ratio. 

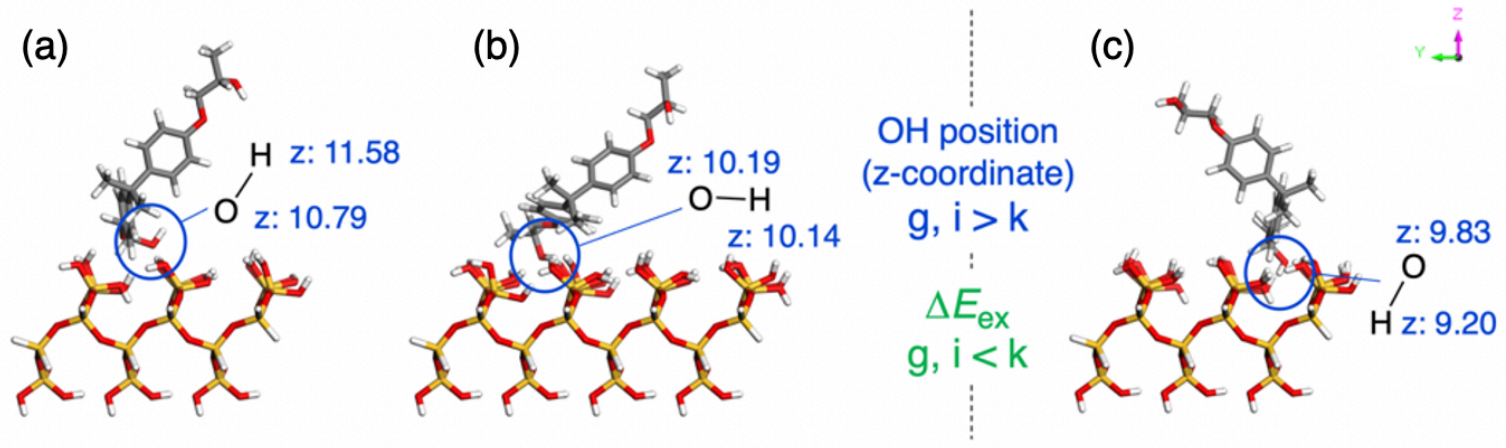

Figure S12. The $z$-coordinates of the terminal $\mathrm{OH}$ groups of models (a) g, (b) i, and (c) k. The $z$-coordinates of the $\mathrm{O}$ and $\mathrm{H}$ atoms are shown after " $z$ :", in $\AA$.

\section{References}

${ }^{1}$ Klier, K.; Shen, J. H.; Zettlemoyer, A. C. Water on Silica and Silicate Surfaces. I. Partially Hydrophobic Silicas. J. Phys. Chem. 1973, 77, 1458-1465.

${ }^{2}$ Higuchi, C.; Tanaka, H.; Yoshizawa, K. Molecular Understanding of the Adhesive Interactions between Silica Surface and Epoxy Resin: Effects of Interfacial Water. J. Comput. Chem. 2019, 40, 164-171.

${ }^{3}$ Downs, R. T.; Palmer, D. C. The pressure behavior of $\alpha$ cristobalite. American Mineralogist 1994, 79, 9-14.

${ }^{4}$ Yoshizawa, K.; Semoto, T.; Hitaoka, S.; Higuchi, C.; Shiota, Y.; Tanaka, H. Synergy of Electrostatic and van Der Waals Interactions in the Adhesion of Epoxy Resin with CarbonFiber and Glass Surfaces. Bull. Chem. Soc. Jpn. 2017, 90, 500-505.

${ }^{5}$ Kitaura, K.; Ikeo, E.; Asada, T.; Nakano, T.; Uebayasi, M. Fragment Molecular Orbital Method: An Approximate Computational Method for Large Molecules. Chem. Phys. Lett. 1999, 313, 701-706.

${ }^{6}$ Fedorov, D. G.; Kitaura, K. The Importance of Three-Body Terms in the Fragment Molecular Orbital Method. J. Chem. Phys. 2004, 120, 6832-6840.

${ }^{7}$ Fedorov, D. G.; Kitaura, K. Extending the Power of Quantum Chemistry to Large Systems with the Fragment Molecular Orbital Method. J. Phys. Chem. A 2007, 111, 6904-6914. 